\title{
VAC.26 - A new vaccination strategy to induce broad range immunity against influenza by using recombinant influenza virus encoding a murine cytokine
}

Sarah Giarola da Silva Messias ${ }^{1 *}$; Ana Paula de Faria Gonçalves ${ }^{1}$; Ianca Évelin Silva de Paula ${ }^{1}$; Lídia Paula Faustino'; Marcelo Antônio Pascoal Xavier ${ }^{1}$; Márcio Sobreira Silva Araújo; Alexandre de Magalhães Vieira Machado ${ }^{1}$.

1 Fiocruz/CPqRR.

Introduction: Influenza is an important public health problem due to its high transmissibility, the ability to cause serious illness and pandemic potential. Immunization is the main strategy to reduce the impact of influenza infections. However, the current vaccines are unable to confer broad range protection against other FLU isolates and subtypes. Therefore, is important to seek alternative vaccine approaches able to induce the heterosubtypic and long-term memory responses. In this context, the cytokine IL-Z (encoded) is an important target in the study of new vaccine adjuvants, since it is associated with homeostasis and cell survival.

Objective: This work aims to evaluate the role of IL-Z cytokine in infection and immunization against influenza virus in murine model.

Methodology: To this end, by using the plasmid driven reverse genetics techniques, we generated a defective recombinant influenza virus (FluIL-Z) encoding the murine IL- $Z$ sequence. After generation of the viral particles, the stocks were produced by cloning in cell culture and characterized by PCR, ELISA, sequencing and titration. Therefore, this virus was used for intranasal immunization of mice (License number: LW-7/17) and evaluated in the co-infection with a $\mathrm{H} 1 \mathrm{~N} 1$ wild-type virus and in the heterosubtipic protection after challange with $\mathrm{H} 3 \mathrm{~N} 2$ virus.

Results: Our results demonstrate that the FluIL-Z is completely safe to mice and was able to induce the expression of high levels of IL-Z cytokine in the cell culture supernatant. In mice infected with the construct, the IL-Z cytokine peak occurs in $24 \mathrm{~h}$ and $48 \mathrm{~h}$ in the lungs and bronchoalveolar lavage, respectively. In addition, mice co-infected with wild-type virus and recombinant virus showed better recovery from the disease and mortality is lower in this group. Finally, FluIL-Z conferred long-term heterosubtypic protection to animals previously immunized and challenged after 30 and 75 days with an influenza virus of another subtype.

Conclusion: In conclusion, our results suggest that FluIL-Z is a promising tool and may support the development of new and more effective immunization strategies against influenza in the future.

Keywords: Influenza vaccines; Recombinant viruses; Reverse genetics 